\title{
The edge of the wedge theorem for separately holomorphic functions with singularities
}

\author{
LuCA BARACCO AND Giuseppe Zampieri
}

\begin{abstract}
For a function that is defined and continuous on $\mathbb{R}^{n}$ except from a $C^{1}$-hypersurface $V \subset \mathbb{R}^{n}$ and that extends as a holomorphic function separately in each complex direction $z_{j}=x_{j}+\mathrm{i} y_{j}$ to $y_{j}>0$, jointly continuous up to $\mathbb{R}^{n} \backslash V$, we prove simultaneous holomorphic extension to the domain $\left\{z=x+\mathrm{i} y \in \mathbb{C}^{n}: y_{j}>0\right.$ for any $\left.j\right\}$ provided that the conormal $v=v_{x}$ to $V$ at any $x \in V$ satisfies $v_{j}>0$ (or $v_{j}<0$ ) for any $j$. This is a generalization of the AjrapetyanHenkin "edge of the wedge theorem" [2] where singularities are not allowed that is $V=\emptyset$. Our statement has also a local variant and, moreover, applies to functions that are defined, when $y_{j}=0$ for any $j$, only on one side of $V$. There is a great amount of work that has been done on the problem of joint analyticity of separately holomorphic functions based on the method of the "pluripotential theory" whose use was initiated by Siciak. In absence of singularities, that is for $V=\emptyset$, we quote among others $[5,16-19]$; in case of $V \neq \emptyset$ analytic, we refer to [10,14]. Also, the above extension principle, in its formulation with a set of singularities $V$, has interesting applications to the range characterization of the exponential Radon transform (cf. $[1,8,12]$ ).
\end{abstract}

\section{Introduction}

The classical "edge of the wedge theorem" by Ajrapetyan-Henkin states that a continuous function $f$ on $\mathbb{R}^{n}$ that extends holomorphically in each $z_{j}=x_{j}+\mathrm{i} y_{j}$ to $y_{j}>0$, jointly continuous up to $y_{j}=0$, extends in fact to the (convex) wedge of $\mathbb{C}^{n}$ defined by $y_{j}>0$ for any $j$. We generalize this statement in the following sense. Let $V \subset \mathbb{C}^{n}$ be a real $C^{1}$ hypersurface of $\mathbb{C}^{n}$ and $f$ be a continuous function in $\mathbb{R}^{n} \backslash V$ that extends holomorphically in each $z_{j}$ to $y_{j}>0$ continuous up to $y_{j}=0$ for any $j$ on $\mathbb{R}^{n} \backslash V$. Then $f$ extends to the quadrant defined by $y_{j}>0$ for any $j$ if we assume that the conormal $v$ to $V$ at any point of $V$ satisfies $v_{j}>0$ (or $v_{j}<0$ ) for any $j$. There is also a local variant of the classical edge of the wedge theorem. For suitable constants 
$c_{1}, c_{2}$, if $f$ is defined in the ball $B\left(x, c_{1} \epsilon\right)$ of center $x$ and radius $c_{1} \epsilon$ and extends holomorphically in each $z_{j}$ to $\left\{y_{j}: 0<y_{j}<c_{2} \epsilon\right\}$, continuous up to $y_{j}=0$ for any $j$, then $f$ extends to $\left\{z: x \in B(x, \epsilon), \sum_{j=1}^{n} y_{j}<\epsilon, y_{j}>0\right\}$. (Indeed we can take $c_{2}=n+1$.) Let $\Omega$ be a domain of $\mathbb{R}^{n}$ with $C^{1}$ boundary. We prove that if over a compact subset of the boundary $K \subset \subset \partial \Omega$ all conormals $v$ to $\Omega$ satisfy $v_{j}>0$ (or $v_{j}<0$ ) for any $j$, then for suitable $c_{1}$ $c_{2}$ and $\epsilon_{0}$, for any $\epsilon<\epsilon_{0}$, and for any point $x \in K, f$ extends holomorphically to $\left\{z=x+\mathrm{i} y: x \in \Omega \cap B(x, \epsilon), \sum_{j=1}^{n} y_{j}<\epsilon\right\}$ when it is supposed to be continuous up to $y_{j}=0$ for any $j$ only on $\Omega \cap B\left(x, c_{1} \epsilon\right)$ instead of the whole $B\left(x, c_{1} \epsilon\right)$.

There is a wide literature about separate analyticity and the so-called "Hartogs phenomenon." The most common approach is based on the "pluripotential theory" due to Siciak $[17,18]$ whose original idea goes back to the celebrated Phragmén-Lindelöf principle or the Bochner's tube theorem (see, e.g., [13] for a local version). The general purpose is to describe the envelop of holomorphy $\hat{X}$ of a set of the type $X=\left(D_{1} \times A_{2} \times A_{3} \times \cdots\right) \cup\left(A_{1} \times D_{2} \times\right.$ $\left.A_{3} \times \cdots\right) \cup \ldots$ where the $D_{j}$ 's are domains of $\mathbb{C}$ and the $A_{j}$ 's are "polar" subsets that is 0 -sets of subharmonic functions $\varphi_{j}$ with $D_{j}$ defined by $\varphi_{j}<1$ : the envelop $\hat{X}$ is the set described by $\sum_{j} \varphi_{j}<1$. In this context, we refer to the papers by Bernstein [5], Siciak [16,17], Zaharjuta [19], Nguyen and Zeriahi [11]. These results were further extended to some cases of non-polar sets $A_{j}$ by Shiffman [15]. Also, there are some variants of the above extension problem in which $f$ is defined outside an analytic subset $V$ of $X: f$ is then holomorphic in $\hat{X} \backslash V^{\mathbb{C}}$ where $V^{\mathbb{C}}$ denotes the complexification of $V$. This result is stated in [16] in case $V$ is the 0 set of a polynomial $P$ and was further extended by Jarnicki and Pflug [10] and Pflug and Nguyen [14] for general analytic sets $V$.

In the applications, of primary interest is the simple case of $X=$ $((\mathbb{R} \times \mathbb{C}) \cup(\mathbb{C} \times \mathbb{R})) \backslash \Delta$ where $\Delta$ is the diagonal of $\mathbb{R}^{2}$ for which our theory provides a particularly simple and geometrically meaningful proof. This situation was treated by Aguilar, et al. [1], Ehrenpreis [8] and Öktem [12], and more recently by Baracco [3]. By the holomorphic extension property which applies to this situation, one sees (cf. the subsequent Appendix) that a function on $\mathbb{R}^{2} \backslash B_{\mu}$, the complement of the ball of radius $\mu$, which is separately entire when restricted to any ray tangent to $\partial B_{\mu}$, is indeed an entire function on $\mathbb{C}^{2}$. From this statement one gets a beautiful characterization of the range of the Radon transform of $C_{c}^{\infty}$ functions in $\mathbb{R}^{2}$. This is described as the collection of all functions separately entire in the above sense and which have in addition the Paley-Wiener exponential growth estimates. 


\section{The generalization of the "edge of the wedge" Theorem}

We use the following notations

$$
\begin{gathered}
\Gamma_{\epsilon}=((0, \epsilon) \times\{0\} \times \cdots) \cup(\{0\} \times(0, \epsilon) \times\{0\} \times \cdots) \cup \cdots \\
\hat{\Gamma}_{\epsilon}=\text { the convex hull of } \Gamma_{\epsilon}
\end{gathered}
$$

Let $\Omega$ be a domain of $\mathbb{R}^{n}$ with $C^{1}$ boundary. We assume in the first part of our discussion that $\Omega$ seats on one side of its boundary. For a point $x \in \partial \Omega$, we denote by $v=v_{x}$ the conormal to $\Omega$ at $x$.

Theorem 2.1. Let $K$ be a compact subset of $\partial \Omega$ and assume that $v_{x} \in \pm \hat{\Gamma}$ for any $x \in K$. There are then $\epsilon_{0}, c_{1}, c_{2}$ such that for any $\epsilon<\epsilon_{0}$, for any $x \in K$ and for any continuous function $f$ on $\Omega \cap B\left(x, c_{1} \epsilon\right)$ which extends as a separately holomorphic function to $B\left(x, c_{1} \epsilon\right)+\mathrm{i} \Gamma_{c_{2} \epsilon}$, continuous up to $\Omega \cap B\left(x, c_{1} \epsilon\right)$, extends holomorphically to $(\Omega \cap B(x, \epsilon))+\mathrm{i} \hat{\Gamma}_{\epsilon}$.

The proof will be given in Section 3. We make now some remarks and discuss some applications and complements.

Remark 2.1. By the classical edge of the wedge theorem of [2], $f$ first extends to a neighborhood of $\Omega \cap B\left(x, c_{1} \epsilon\right)$ in the sector $\left\{y_{j}>0\right.$ for any $\left.j\right\}$; next, by the theorem of Hanges-Treves [9], this extension propagates along the $z_{j}$-planes to a neighborhood of $B\left(x, c_{1} \epsilon\right)+\mathrm{i} \Gamma_{c_{2} \epsilon}$ in the sector $\left\{z \in \mathbb{C}^{n}\right.$ : $y_{j}>0$ for any $\left.j\right\}$. The initial extension described above is founded on a construction of a family of discs of the type of our subsequent proof in addition to the approximation theorem by Baouendi-Treves [4]. Our result consists in starting from this initial domain of holomorphic extension and in improving it to the final domain $(\Omega \cap B(x, \epsilon))+\mathrm{i} \hat{\Gamma}_{\epsilon}$. Its proof is completely self-contained; also, it relies on the continuity principle instead of the approximation theorem.

Remark 2.2. The proof of Theorem 2.1 shows that if for any $x \in \partial \Omega$ the conormal $v_{x}$ is contained in a proper subcone of $\hat{\Gamma}$, there are then $\epsilon_{0}, c_{1}, c_{2}$ such that separately holomorphic functions on $B\left(x, c_{1} \epsilon\right)+\mathrm{i} \Gamma_{c_{2} \epsilon}$ (with $x \in \partial \Omega$ and $\left.\epsilon<\epsilon_{0}\right)$, continuous up to $\Omega \cap B\left(x, c_{1} \epsilon\right)$, extend to $(\Omega \cap B(x, \epsilon))+\mathrm{i} \hat{\Gamma}_{\epsilon}$.

In the statements which follow we can allow any size in the $y$-plane for the sets of holomorphic extension. Also, the shrinking in some parameters is not related to the geometry of $\Omega$. Hence we disregard it and denote by a common 
notation $\Gamma$ or $\hat{\Gamma}$ the cones as in (2.1) and (2.2) without specifying their truncatures. These cones can possibly be infinite, that is, non-truncated.

Theorem 2.2. Let $\Omega$ be a domain of $\mathbb{R}^{n}$ with $C^{1}$-boundary such that for any $x \in \partial \Omega$ the conormal $v=v_{x}$ satisfies $v \in \pm \hat{\Gamma}$. Then any separately holomorphic function $f$ on $\mathbb{R}^{n}+i \dot{\Gamma}$, continuous up to $\Omega$ extends holomorphically to $\Omega+\mathrm{i} \hat{\Gamma}$.

The proof follows in Section 3. Remark that in case $\partial \Omega$ is an analytic hypersurface $V$ defined, say, by $l(x)=0$, the hypothesis $v \in \pm \hat{\Gamma}$ yields $V^{\mathbb{C}} \cap(\Omega+\mathrm{i} \hat{\Gamma})=\emptyset$. The above condition is clearly necessary for the function $f(z)=\frac{1}{l(z)}$, singular along $V^{\mathbb{C}}$, not to be in contrast with Theorem 2.2. Note that in the above statement the condition about the conormal must hold all along $\partial \Omega$; in particular, $\Omega$ must be unbounded.

We change a little from now on our setting and assume that $\Omega=\mathbb{R}^{n} \backslash V$ for a $C^{1}$-hypersurface $V$ of $\mathbb{R}^{n}$. We denote by $M$ the hypersurface of $\mathbb{C}^{n}$ defined by $M=V+\mathrm{i} \mathbb{R}^{n}$. We recall that $M$ is said to be "minimal" at any of its points (in the sense of Trepreau and Tumanov) when it does not contain any germ of complex hypersurface. We also recall that this situation characterizes the property that holomorphic functions extend from one of the two sides of $M$ to a full neighborhood of any of its boundary points. We begin by proving that the case when $M$ is non-minimal is exceptional according to the following statement which can be found in the literature, e.g., in [7].

Proposition 2.1. Let $\gamma$ be an open complex hypersurface in $M$, and denote by $\pi: \mathbb{C}^{n} \rightarrow \mathbb{R}^{n} z \mapsto x$ the projection. Then $\gamma$ is a complex hyperplane and $\pi(\gamma)$ is an open piece of $V$.

Proof. Let $T^{\mathbb{C}} M=T M \cap \mathrm{i} T M$ be the complex tangent bundle to $M$ and note that due to the special choice of $M$, we have for any $z_{o}=x_{o}+i y_{o} \in M$ :

$$
T_{z_{\mathrm{o}}}^{\mathbb{C}} M=T_{x_{\mathrm{o}}} V+\mathrm{i} T_{x_{\mathrm{o}}} V
$$

A CR curve on $M$ is an integral curve of a section of $T^{\mathbb{C}} M$ and a CR orbit is a union of piecewise $C^{1}$ smooth $\mathrm{CR}$ curves. Note that $\gamma$ is necessarily a $\mathrm{CR}$ orbit whence in particular any $\mathrm{CR}$ curve isssued from its points must lie in $\gamma$. Also, for $z_{\mathrm{o}} \in \gamma$, we must have

$$
\begin{aligned}
T_{z_{\mathrm{o}}} \gamma & =T_{z_{\mathrm{o}}}^{\mathbb{C}} M \\
& =T_{x_{\mathrm{o}}} V+\mathrm{i} T_{x_{\mathrm{o}}} V
\end{aligned}
$$


due to (2.3). But for any fixed $y \in T_{x_{\mathrm{o}}} V$ the line $z_{o}+$ ity for $t \in \mathbb{R}$ is a CR curve and therefore it is contained in $\gamma$. It follows that $\gamma($ resp. $V$ ) is a complex (resp. real) plane and indeed $\gamma=T_{x_{\mathrm{o}}} V+\mathrm{i} T_{x_{\mathrm{o}}} V\left(\right.$ resp. $V=T_{x_{\mathrm{o}}} V$ in a neighborhood of $x_{\mathrm{o}}$ ).

Note that at any point $z_{\mathrm{o}}$ in the closure of $\gamma$, but not in $\gamma, M$ is minimal. Otherwise there should exist a germ of a complex hypersurface through $z_{\mathrm{o}}$ which would necessarily glue with $\gamma$.

Theorem 2.3. (i) Let $K$ be a compact subset of $V$ and assume that $v_{x} \in$ $\pm \hat{\Gamma}$ for any $x \in K$. Then there are $\epsilon_{0}, c_{1}, c_{2}$ such that for any $\epsilon<\epsilon_{0}$, for any $x \in K$ and for any continuous function $f$ on $\left(\mathbb{R}^{n} \backslash V\right) \cap B\left(x, c_{1} \epsilon\right)$ which extends as a separately holomorphic function to $B\left(x, c_{1} \epsilon\right)+\mathrm{i} \Gamma_{c_{2} \epsilon}$, continuous up to $\left(\mathbb{R}^{n} \backslash V\right) \cap B\left(x, c_{1} \epsilon\right)$, extends holomorphically to $B(x, \epsilon)+\mathrm{i} \hat{\Gamma}_{\epsilon}$.

(ii) Let $v_{x} \in \pm \hat{\Gamma}$ for any $x \in V$. Then any $f$ separately holomorphic in $\mathbb{R}^{n}+\mathrm{i} \Gamma$, continuous up to $\mathbb{R}^{n} \backslash V$, extends to $\mathbb{R}^{n}+\mathrm{i} \hat{\Gamma}$, continuous up to $\mathbb{R}^{n} \backslash V$.

Proof. The proof for the two cases, local and global, is the same so we do not distinguish the related notations. We first extend $f$ to $\left(\mathbb{R}^{n} \backslash V\right)+\mathrm{i} \hat{\Gamma}$ by the aid of Theorem 2.2. Next, we can have two possibilities. The hypersurface $V+\mathrm{i} \mathbb{R}^{n}$ is minimal; hence $f$ extends from one side of $V+\mathrm{i} \hat{\Gamma}$ and since it extends from both sides at $V+\mathrm{i} \Gamma$, then $f$ extends to $\mathbb{R}^{n}+\mathrm{i} \hat{\Gamma}$. The alternative is that $V$ is a real plane defined, say, by $l(x)=0$. Hence $V+$ $\mathrm{i} \hat{\Gamma}$ is foliated by the complex planes defined by $l(z)=\mathrm{i} t$ for $t \in \mathbb{R}$ which all intersect $V+\mathrm{i} \Gamma$. Thus holomorphicity at $V+\mathrm{i} \Gamma$ propagates all over $V+\mathrm{i} \hat{\Gamma}$.

We further specialize our discussion and consider functions which extend as separately holomorphic to both sides $y_{j}>0$ and $y_{j}<0$. We use the notations

$$
\Sigma:=(\mathbb{R} \times\{0\} \times \cdots) \cup(\{0\} \times \mathbb{R} \times\{0\} \times \cdots) \cup \cdots, \quad \dot{\Sigma}=\Sigma \backslash\{0\} .
$$

From now on all our proofs and statements are the same for the local and the global cases so we do not distinguish the related notations. In particular, $\Sigma$ will be truncated or not without specification. By collecting the conclusions of Theorem 2.1 slightly modified, with those of Proposition 2.1, we get the following statement which is largely contained in [3]. 
Theorem 2.4. Assume that

(2.5) for any $x \notin V$ there is a "quadrant", say $\hat{\Gamma}$, s.t. $(x+\hat{\Gamma}) \cap V=\emptyset$.

Then any function $f$ on $\mathbb{R}^{n} \backslash V$ which extends holomorphically to $\left(\mathbb{R}^{n} \backslash V\right)+$ $\mathrm{i} \Sigma$ extends in fact to the whole $\mathbb{C}^{n}$ unless $V$ is a plane in which case $f$ extends to $\mathbb{C}^{n} \backslash V^{\mathbb{C}}$.

Proof. First, we extend $f$ to $\left(\mathbb{R}^{n} \backslash V\right)+i \mathbb{R}^{n}$. In fact, because of our assumption (2.5), we can adapt the proof of Theorem 2.1 and manage to handle the points $y$ not only for $y \in \pm \hat{\Gamma}$ but for all occurrences $y \in \mathbb{R}^{n}$. (It is easy to make the right choice of the $\eta_{j}$ 's according to all possible occurrences of signs.)

Next, there may be two possibilities. Either $V+\mathrm{i} \mathbb{R}^{n}$ is minimal and therefore $f$ extends from one side of $M=V+i \mathbb{R}^{n}$ and since it extends from both sides at $V+\mathrm{i} \dot{\Sigma}$, then it extends in fact from both sides all over $M$. The other case, according to Proposition 2.1, is that $V$ is a real plane defined, say, by $l(x)=0$. Then $M$ is foliated by the family of complex planes with parameter $t$ defined by $l(z)=\mathrm{it}$ and these have non-empty intersection with $V+\mathrm{i} \dot{\Sigma}$ apart from the plane $V^{\mathbb{C}}$ itself (which corresponds to the choice $t=0$ ). Since $f$ was already known to be holomorphic on $V+i \dot{\Sigma}$, the theorem follows.

\section{Proof of Theorems 2.1 and 2.2}

We denote by $z$ the variable in $\mathbb{C}^{n}$ and by $\zeta$ or $\tau$ that in $\mathbb{C}$. We denote by $\Delta$ the unit disc in $\mathbb{C}$ and by $\partial \Delta$ its boundary parametrized by $\mathrm{e}^{\mathrm{i} \theta}$ or $\mathrm{e}^{\mathrm{i} \varphi}$ for $\theta$ and $\varphi$ in $[0,2 \pi]$. For a function $\eta$ on $[0,2 \pi]$ or $\partial \Delta$, we write equivalently $\eta(\theta)$ or $\eta(\zeta)$ under the above identification. For a real function $\eta$, we define the Hilbert transform $T \eta$ as the function on $\partial \Delta$, determined up to real constants, such that $\eta+\mathrm{i} T \eta$ extends holomorphically from $\partial \Delta$ to $\Delta$. We define

$$
\chi_{\varphi}(\theta)=\frac{\sin (\varphi-\theta)}{2 \pi(1-\cos (\varphi-\theta))} .
$$

It is easy to prove that, for $\tau=\mathrm{e}^{\mathrm{i} \varphi}$ and $\zeta=\mathrm{e}^{\mathrm{i} \theta}$ we have

$$
\begin{aligned}
T \eta(\tau) & =\operatorname{Im}\left(\frac{1}{2 \pi \mathrm{i}} \int_{+\partial \Delta} \frac{\zeta+\tau}{\zeta-\tau} \eta(\zeta) \frac{d \zeta}{\zeta}\right) \\
& =\operatorname{Im}\left(\frac{1}{2 \pi} \int_{0}^{2 \pi} \frac{\mathrm{e}^{\mathrm{i} \theta}+\mathrm{e}^{\mathrm{i} \varphi}}{\left.\mathrm{e}^{\mathrm{i} \theta}-\mathrm{e}^{\mathrm{i} \varphi} \eta(\theta) d \theta\right)}\right. \\
& =\int_{0}^{2 \pi} \chi_{\varphi}(\theta) \eta(\theta) d \theta
\end{aligned}
$$


Note that the harmonic extension of $T \eta$ from $\partial \Delta$ to $\Delta$ is explicitly described by the first line of (3.1). In particular

$$
T \eta(0)=0
$$

We also point out an important feature of $\chi$ that will play an essential role in the following:

$$
\chi_{\varphi}(\theta)=\frac{1}{\pi(\theta-\varphi)}+O(|\theta-\varphi|) .
$$

We denote by $C^{1, \alpha}(\partial \Delta)$ for $0<\alpha<1$ the space of functions whose first derivatives are $\alpha$-Hölder continuous. It is well known (cf. [6]) that $T$ is a bounded operator when acting on $C^{1, \alpha}$ (the fractional regularity being essential for this conclusion).

For a point $x$ in $\mathbb{R}^{n}$, we consider the discs

$$
A_{x}=x+A(\tau) \text { with } A: \bar{\Delta} \rightarrow \mathbb{C}^{n}, A(\tau)=x(\tau)+\mathrm{i} y(\tau), x(0)=0
$$

these are analytic in $\Delta$ and $C^{1, \alpha}$ up to $\partial \Delta$. We denote by the same symbol $A_{x}$ both the parametrization maps and their ranges.

Proof of Theorem 2.1. It suffices to prove that we may find $\epsilon_{0}, c_{1}, c_{2}$, depending on $K \subset \subset \partial \Omega$ such that for $x \in K$ and for $x^{\prime} \in \Omega \cap B(x, \epsilon)$ with $\epsilon<\epsilon_{0}$, there exists a family of $\operatorname{discs} A_{x^{\prime}}$ which satisfy the following claims:

(i) Each disc $A_{x^{\prime}}$ is "attached" to $S:=\left(\Omega \cap B\left(x, c_{1} \epsilon\right)\right) \cup\left(B\left(x, c_{1} \epsilon\right)+\right.$ $\left.\mathrm{i} \Gamma_{c_{2} \epsilon}\right)$ in the sense that $A_{x^{\prime}}(\partial \Delta) \subset S$.

(ii) The set of centers $\left\{A_{x^{\prime}}(0)\right\}$ fills a region which contains $x^{\prime}+\mathrm{i} \hat{\Gamma}_{\epsilon}$.

(iii) Each disc $A_{x^{\prime}}$ can be contracted to the "limit" disc $\left\{x^{\prime}\right\}$ through a family of attached discs.

If we succeed in proving these claims, then the conclusion follows at once. In fact, let $f$ be as in the statement of Theorem 2.1. We recall from Remark 2.1 that $f$ extends holomorphically to an initial domain, a neighborhood of $S$ intersected with the sector $\left\{z \in \mathbb{C}^{n}: y_{j}>0\right.$ for any $\left.j\right\}$. Since each disc $A_{x^{\prime}}$ is contractible to $x^{\prime} \in \Omega$, and has its boundary in $S$, then if we perturb a little this family by pushing it inside the sector $\left\{z \in \mathbb{C}^{n}: y_{j}>0\right.$ for any $\left.j\right\}$, we conclude, by the continuity principle, that $f$ extends from the boundaries to the whole discs, hence to the region $x^{\prime}+\mathrm{i} \hat{\Gamma}_{\epsilon}$ swept out by their centers. Thus, let us prove the three above claims. We recall that we are denoting by $v=v_{x}$ the normal to $\Omega$ at $x$; we fix a choice of its orientation and assume 
that it points inward $\Omega$. We assume throughout the proof that $v \in \hat{\Gamma}$ (the symmetric case $v \in-\hat{\Gamma}$ being treated similarly). We note that for $\epsilon$ and $\epsilon^{\prime}$ small enough we have

$$
\left|x^{\prime}-x\right|<\epsilon, x^{\prime \prime} \cdot v_{x} \geq \epsilon^{\prime}\left|x^{\prime \prime}\right|,\left|x^{\prime \prime}\right|<\epsilon^{\prime} \text { implies } x^{\prime}+x^{\prime \prime} \in \Omega .
$$

We take $\lambda_{j} \geq 0$ with $\sum_{j} \lambda_{j} \leq \epsilon$ and give an increasing order to the numbers $\lambda_{j} v_{j}$; to fix notations we assume

$$
\lambda_{1} v_{1} \leq \lambda_{2} v_{2} \leq \cdots \leq \lambda_{n} v_{n}
$$

We decompose the interval $[0,2 \pi]$ in $n$ intervals by setting $\varphi_{0}=0, \varphi_{j}=$ $\frac{j \pi}{2(n-1)}$ for $j \leq n-1, \varphi_{n}=2 \pi$ and finally define $I_{j}=\left[\varphi_{j-1}, \varphi_{j}\right]$. We recall the kernel $\chi_{\varphi}(\theta)=\frac{\sin (\varphi-\theta)}{2 \pi(1-\cos (\varphi-\theta))}$; we note that

$$
\chi_{\varphi_{j}}(\theta)<0 \text { for any } j=0, \ldots, n-1 \text { and for any } \theta \in\left(\frac{\pi}{2}, \pi\right) \text {. }
$$

(To treat the case $v \in-\hat{\Gamma}$ we have to notice, instead of (3.6), that

$$
\left.\chi_{\varphi_{j}}(\theta)>0 \text { for any } j=0, \ldots, n-1 \text { and for any } \theta \in\left(\frac{3}{2} \pi, 2 \pi\right) .\right)
$$

We choose positive real smooth functions $\eta_{j}$ such that

$$
\begin{gathered}
\operatorname{supp}\left(\eta_{j}\right) \subset\left[\varphi_{j-1}, \varphi_{j}\right] \\
\eta_{j} \in C^{1, \alpha} \text { and } \eta_{j}=0 \text { exactly at } \theta=\varphi_{j-1}, \varphi_{j} . \\
\frac{1}{2 \pi} \int_{\varphi_{j-1}}^{\varphi_{j}} \eta_{j} d \theta=1 \text { for any } j \leq n-1, \\
\frac{1}{2 \pi} \int_{\frac{\pi}{2}}^{\pi} \eta_{n} d \theta=1-\epsilon^{\prime}, \frac{1}{2 \pi} \int_{\pi}^{2 \pi} \eta_{n} d \theta=\epsilon^{\prime} . \\
\eta_{j} \leq c_{2} .
\end{gathered}
$$

Clearly we have $\eta_{j}^{\prime}=0$ at $\theta=\varphi_{j-1}, \varphi_{j}$. Note here that to have (3.10) fulfilled by any $\eta_{j}$ for $j=1, \ldots, n-1$, we need in (3.11) a constant $c_{2}=c_{2}(n)>$ $4(n-1)$ (in particular this constant goes to $+\infty$ as $n \rightarrow+\infty$ ) whereas $\eta_{n}$ may be estimated by a fixed constant $c_{2}>4$. It is also clear that

$$
\left|T \eta_{j}(\theta)\right|<c_{1} \quad \text { for any } j \text { and for any } \theta \in[0,2 \pi] \text {. }
$$

Again, $\left|T \eta_{j}\right|<c_{1}(n)$ for any $j=1, \ldots, n-1$ with $c_{1}(n) \rightarrow+\infty$ whereas $\left|T \eta_{n}\right|<c_{1}$ for $c_{1}$ fixed. We further specify our choice of the $\eta_{i}$ 's. We remark 
that $T \eta_{i}\left(\varphi_{j}\right)$ is $>0$ or $<0$ according to $j \geq i$ or $j<i$; this is obvious when $i<n$ or $i=n$ and $j<0$. As for the case $i=n, j=0$, we just have to take $\int_{\pi}^{2 \pi} \eta_{n} d \theta$ small. In particular, we then have $T \eta_{i}\left(\varphi_{0}\right)<0$ for any $i$. If $\eta_{1}$ has been chosen, we take $\eta_{2}$ that satisfies the above requirements and also such that $T \eta_{1}\left(\varphi_{2}\right)<-T \eta_{2}\left(\varphi_{2}\right)-\epsilon^{\prime}$. We then proceed by induction. If $\eta_{1}, \ldots, \eta_{j}$ have been taken, we choose $\eta_{j+1}$ such that

$$
\sum_{i \leq j} T \eta_{i}\left(\varphi_{j+1}\right)<-T \eta_{j+1}\left(\varphi_{j+1}\right)-\epsilon^{\prime}
$$

In other terms $\sum_{i<j+1} T \eta_{i}\left(\varphi_{j}\right)<-\epsilon^{\prime}$ and so for the full family of the $\eta_{i}$ 's we have $\sum_{i \leq n} T \eta_{i}\left(\varphi_{j}\right)<-\epsilon^{\prime}$ for any $j=0, \ldots, n-1$ due to $T \eta_{i}\left(\varphi_{j}\right)<0$ for any $i \geq j+1$. (In case $v \in-\hat{\Gamma}$, we have to make a different choice of the $\eta_{i}$ 's. First, we have to take $\eta_{n}$ satisfying, instead of (3.10), $\frac{1}{2 \pi} \int_{\frac{3}{2} \pi}^{2 \pi} \eta_{n} d \theta=1-\epsilon^{\prime}$, $\frac{1}{2 \pi} \int_{\frac{\pi}{2}}^{\frac{3}{2} \pi} \eta_{n} d \theta=\epsilon^{\prime}$. Next, we note that $T \eta_{i}\left(\varphi_{n-1}\right)>0$ for any $i$. Thus, if $\eta_{n-1}$ has already been taken, we choose $\eta_{n-2}$ such that $T \eta_{n-2}\left(\varphi_{n-2}\right)>$ $T \eta_{n-1}\left(\varphi_{n-2}\right)+\epsilon^{\prime}$. Reasoning by induction as above, we end up with a family of $\eta_{i}$ 's which satisfy $\sum_{i \leq n} T \eta_{i}\left(\varphi_{j}\right)>\epsilon^{\prime}$ for any $j=0, \ldots, n-1$.)

Let $e_{j}$ be the unit coordinate vectors. For $x^{\prime} \in \Omega \cap B(x, \epsilon)$ and $\lambda \in \hat{\Gamma}_{\epsilon}$ we take $\eta_{j}$ chosen as above (depending on $x^{\prime}$ and $\lambda$ ), set $\eta:=\sum_{j} \lambda_{j} \eta_{j} e_{j}$, and consider the disc

$$
A_{x^{\prime}}=\left(x^{\prime}-T \eta\right)+\mathrm{i} \eta
$$

(In the case $v \in-\hat{\Gamma}$ we just define $A_{x^{\prime}}=\left(x^{\prime}+T \eta\right)-\mathrm{i} \eta$.) We recall that $T \eta(0)=0$ and remark that $\eta(0)=\frac{1}{2 \pi} \sum_{j} \lambda_{j} e_{j} \int_{0}^{2 \pi} \eta_{j} d \theta=\sum_{j} \lambda_{j} e_{j}$; hence we have for the centers

$$
A_{x^{\prime}}(0)=x^{\prime}+\mathrm{i} \sum \lambda_{j} e_{j}
$$

Hence (ii) is fulfilled and (iii) is also evident by letting $\lambda_{j} \rightarrow 0$ for any $j$. We notice that $\partial A_{x^{\prime}} \backslash \mathbb{R}^{n} \subset \mathbb{R}^{n}+\mathrm{i} \Gamma$ since we are supposing that at no point $\theta \in[0,2 \pi]$ two of the $\eta_{i}$ 's are simultaneously non-vanishing. We also have

$$
\partial A_{x^{\prime}} \backslash \mathbb{R}^{n} \subset B\left(x, c_{1} \epsilon\right)+\mathrm{i} \Gamma_{c_{2} \epsilon} \text { due to (3.12) and (3.11). }
$$

We want to prove now that

$$
\partial A_{x^{\prime}} \cap \mathbb{R}^{n} \subset \Omega \cap B\left(x, c_{1} \epsilon\right) .
$$


According to (3.4), it suffices to prove that $T \eta\left(\varphi_{j}\right) \cdot v<-\epsilon^{\prime}\left|T \eta\left(\varphi_{j}\right)\right|$ for any $j$. But in fact, by (3.13), $\sum_{i \leq n} T \eta_{i}\left(\varphi_{j}\right) \cdot v<-\epsilon^{\prime}$ and hence by $(3.5) \sum_{i \leq n}$ $T \eta_{i}\left(\varphi_{j}\right) \lambda_{i} v_{i}<-\epsilon^{\prime}|\lambda|$. Since it is trivial that $|\lambda| \simeq\left|T \eta\left(\varphi_{j}\right)\right|$, we get the conclusion (for a different $\epsilon^{\prime}$ ). Thus $x^{\prime}-T \eta\left(\varphi_{j}\right) \in \Omega$ for any $j$ which implies (3.17). (The end of the proof for the case $v \in-\hat{\Gamma}$ is similar and only requires to switch from $\eta$ to $-\eta$.) This concludes the proof of Theorem 2.1.

Proof of Theorem 2.2. We assume that $\Gamma$ and $\hat{\Gamma}$ are non-truncated during the proof. By Theorem 2.1, for any $x \in \partial \Omega$ and for a suitably small $\epsilon, f$ extends to $(B(x, \epsilon) \cap \Omega)+\mathrm{i} \hat{\Gamma}_{\epsilon}$. By a finite covering argument, for any $K \subset \subset$ $\mathbb{R}^{n}$ and for some $\epsilon, f$ extends to $(K \cap \Omega)+\mathrm{i} \hat{\Gamma}_{\epsilon}$. Now, we prove that for any $K^{\prime} \subset \subset \mathbb{R}^{n}$, for any $t>0$ large, and for suitable $\epsilon$, we can find a family of $\operatorname{discs} A_{x^{\prime}}, x^{\prime} \in K^{\prime} \cap \Omega$ such that

$$
\left\{\begin{array}{l}
A_{x^{\prime}} \text { is attached to } \Omega \cup\left((K \cap \Omega)+\mathrm{i} \hat{\Gamma}_{\epsilon}\right) \cup\left(\mathbb{R}^{n}+\mathrm{i} \Gamma\right) \\
\text { The centers } A_{x^{\prime}}(0) \text { fill }\left(K^{\prime} \cap \Omega\right)+\mathrm{i} \hat{\Gamma}_{t}
\end{array}\right.
$$

Again, the family of discs is contractible to the points $x^{\prime}$. If we succeed in proving these claims, then by the same argument as in the proof of Theorem 2.1, $f$ extends to $\left(K^{\prime} \cap \Omega\right)+\mathrm{i} \hat{\Gamma}_{t}$, hence to the whole $\Omega+\mathrm{i} \hat{\Gamma}$ by letting $K^{\prime} \rightarrow \mathbb{R}^{n}$ and $t \rightarrow+\infty$. We still describe analytic discs by $A_{x^{\prime}}=$ $\left(x^{\prime}-T \eta\right)+\mathrm{i} \eta$, and make a suitable choice of $\eta$. We take $\eta=\left(\eta_{j}\right)$ such that

(a) $x^{\prime}-\left.T \eta\right|_{\partial \Delta} \in K$ for any $x^{\prime} \in K^{\prime} \cap \Omega$;

(b) $|T \eta|$ is arbitrarily close to 0 at the points where $\eta_{j}=0$ for any $j$ and $T \eta \cdot v \geq \epsilon^{\prime}|T \eta|$; in particular $x^{\prime}-T \eta \in \Omega$ at these points;

(c) either $\eta_{j} \neq 0$ only for one index $j$, or otherwise, $|\eta|<\epsilon$.

We also make our choice so that $\frac{1}{2 \pi} \int_{0}^{2 \pi} \eta_{j} d \theta=1$. To define such a $\eta_{j}$ 's, we divide $[0,2 \pi]$ into disjoint intervals $I_{j}=\left[\varphi_{j-1}, \varphi_{j}\right], j=1, \ldots, n$ of equal length $\frac{2 \pi}{n}$. We want $A_{x^{\prime}}$ map $I_{j}$ into $\mathbb{R}^{n}+i s e_{j}$ for $0<s<1$ except from two neighborhoods of the end-points $\varphi_{j-1}$ and $\varphi_{j}$, with the end-points removed, which are sent to $K+\mathrm{i} \hat{\Gamma}_{\epsilon}$ and from the end-points themselves which are sent to an arbitrary neighborhood of $x^{\prime}$ on $\Omega$ where $f$ extends holomorphically into the sector $\left\{z \in \mathbb{C}^{n}: y_{j}>0\right.$ for any $\left.j\right\}$ according to Remark 2.1. We first take $\eta_{j} \in C^{1, \alpha}$ supported in each $I_{j}$ such that

$$
0 \leq \eta_{j} \leq n+1, \quad \eta_{j}=0 \text { exactly at } \varphi_{j-1} \text { and } \varphi_{j}
$$


and such that $\frac{1}{2 \pi} \int_{0}^{2 \pi} \eta_{j} d \theta=1$. We then take, for any arbitrarily small $\nu$, a first set of small perturbations $\left\{\delta_{j}^{1}\right\}_{j=1, \ldots, n}$, positive, supported near $\varphi_{1}$ from one of the two sides to get compensation of sign and such that

$$
\int \chi_{1} \eta_{j} d \theta+\int \chi_{1} \delta_{j}^{1} d \theta<\nu
$$

This is possible due to (3.3). We also take $\left|\delta_{j}^{1}\right|<\epsilon$ and suppose that $\operatorname{supp}\left(\delta_{j}^{1}\right)$ are so small that $\eta_{1}<\epsilon$ and $\eta_{2}<\epsilon$ over $\operatorname{supp}\left(\delta_{j}^{1}\right)$; this choice brings part of the boundary of $A_{x^{\prime}}$ into the domain of holomorphic extension given by Theorem 2.1. Note that the $\delta_{j}^{1}$ 's are small in $C^{0}$-norm but only bounded in $C^{1}$-norm in order to compensate $\left|T \eta_{j}\right|$. We claim that we can find $\left\{\delta_{j}^{h}\right\}_{j}$ for any $h \leq n$ for any $j \leq n$ such that

$$
\sum_{h \leq n} \int \chi_{k} \delta_{j}^{h} d \theta+\int \chi_{k} \eta_{j} d \theta<\nu \text { for any } k \leq n, \text { for any } j \leq n .
$$

We use induction and assume that for some $m<n$ we have already found a family of perturbations $\left\{\delta_{j}^{h}\right\}$ for any $j \leq n$ for any $h \leq m$ such that

$$
\sum_{h \leq m} \int \chi_{k} \delta_{j}^{h} d \theta+\int \chi_{k} \eta_{j} d \theta<\nu \text { for any } k \leq m, \text { for any } j \leq n
$$

On account of (3.3) we then choose new functions $\delta_{j}^{m+1}$ at the new extremal point $\varphi_{m+1}$ supported near $\varphi_{m+1}$ (and from the suitable side) such that

$$
\int \chi_{m+1} \delta_{j}^{m+1} d \theta+\left(\sum_{h \leq m} \int \chi_{m+1} \delta_{j}^{h} d \theta+\int \chi_{m+1} \eta_{j} d \theta\right)<\nu
$$

still keeping the property, by a choice of small $\delta_{j}^{m+1}$, that for any $k \leq m$ :

$$
\int \chi_{k} \delta_{j}^{m+1} d \theta+\left(\sum_{h \leq m} \int \chi_{k} \delta_{j}^{h} d \theta+\int \chi_{k} \eta_{j} d \theta\right)<\nu
$$

Thus the inductive argument is proved. We take $\lambda_{j} \geq 0$ with $\sum_{j} \lambda_{j}<t$ and set $\eta:=\sum_{j=1, \ldots, n} \lambda_{j}\left(\eta_{j}+\sum_{h=1, \ldots, n} \delta_{j}^{h}\right) e_{j}$. We recall that $T \eta(0)=0$ and remark that $\eta(0)=\sum_{j} \lambda_{j} e_{j} \frac{1}{2 \pi} \int_{0}^{2 \pi}\left(\eta_{j}+\sum_{h} \delta_{j}^{h}\right) d \theta$ is arbitrarily close to 
$\sum_{j} \lambda_{j} e_{j}$; hence we have for the centers

$$
A_{x^{\prime}}(0) \text { is arbitrarily close to } x^{\prime}+\mathrm{i} \sum \lambda_{j} e_{j} .
$$

We recall that $A_{x^{\prime}}$ touches $y_{j}=0$ for any $j$ only at the extremal points $\varphi_{j}$; for these points we have that

$$
\text { " } A_{x^{\prime}}\left(\varphi_{j}\right)=x^{\prime}-\operatorname{T\eta }\left(\varphi_{j}\right) \text { with }\left|T \eta\left(\varphi_{j}\right)\right|<\nu \text { so that } A_{x^{\prime}}\left(\varphi_{j}\right) \in \Omega "
$$

This proves (b). Also, our choice of small $\delta_{j}^{h}$, with support in small neighborhoods of the points $\varphi_{h}$, implies that (c) is fulfilled. We finally prove that for a suitable constant $c$, we have all over $\partial \Delta$ :

$$
|T \eta| \leq c
$$

and hence in particular

" $A_{x^{\prime}}(\theta)$ for any $\theta$ has real part which belongs to $K$ for any $x^{\prime} \in K^{\prime \prime}$

which implies (a). In fact, recall again (3.3). For any fixed $\varphi^{\mathrm{o}} \in[0,2 \pi]$ and for $\kappa$ small let us decompose integration over $[0,2 \pi]$ as $\int_{0}^{2 \pi} \cdot=\int_{0}^{\varphi^{\circ}-\kappa} \cdot+$ $\int_{\varphi^{\circ}+\kappa}^{2 \pi} \cdot+\int_{\varphi^{\circ}-\kappa}^{\varphi^{\circ}+\kappa} \cdot$. If we integrate $\chi_{\varphi^{\circ}} \eta$ using the above decomposition, then the absolute values of the two first integrals are estimated by $c$, and as for the third we have

$$
\begin{aligned}
\left|\int_{\varphi^{\circ}-\kappa}^{\varphi^{\circ}+\kappa} \chi_{\varphi^{\circ}} \eta d \theta\right| & \leq\left|\int_{\varphi^{\circ}-\kappa}^{\varphi^{\circ}+\kappa} \eta\left(\varphi^{\mathrm{o}}\right) \frac{1}{\theta-\varphi^{\mathrm{o}}} d \theta\right|+\left|\int_{\varphi^{\circ}-\kappa}^{\varphi^{\circ}+\kappa} \eta^{\prime}\left(\varphi^{\mathrm{o}}\right) d \theta\right|+O\left(\kappa^{2}\right) \\
& \leq \kappa\left|\eta^{\prime}\left(\varphi^{\mathrm{o}}\right)\right|+O\left(\kappa^{2}\right) .
\end{aligned}
$$

Then (3.20) follows. This completes the proof of Theorem 2.2.

\section{Appendix (Application to Radon transform)}

In the plane $\mathbb{R}^{2}$ we consider the disc $B_{\mu}$ of center 0 and radius $\mu$ and the circle $S_{\mu}=\partial B_{\mu}$. We identify in $\mathbb{R}^{2}$ points $x$ to vectors $v$ by the correspondence $x \mapsto v:=x-0$; we attach the suffix " $\perp$ " to a vector $v$ and write $v^{\perp}$, in order to denote the orthogonal vector of equal length (and with $\left(v, v^{\perp}\right)$ giving the standard orientation). From any point $x \in \mathbb{R}^{2} \backslash B_{\mu}$ we can issue exactly two lines tangent to $S_{\mu}$ at a pair of points $\omega^{\perp}, \sigma^{\perp}$; we can thus write for a 
Edge of the wedge theorem for separately holomorphic functions 339 suitable $t \in \mathbb{R}$

$$
\begin{aligned}
x & =\mu \omega^{\perp}+t \omega \\
& =\mu \sigma^{\perp}-t \sigma .
\end{aligned}
$$

We have the relation

$$
x=\mu \frac{(\omega+\sigma)^{\perp}}{1+\omega \cdot \sigma} .
$$

We denote by $\alpha$ and $\beta$ the arguments of $\omega$ and $\sigma$, respectively, write $\omega=$ $(\cos \alpha, \sin \alpha), \sigma=(\cos \beta, \sin \beta)$, define $\xi:=\operatorname{tg} \frac{\alpha}{2}, \eta=\operatorname{tg} \frac{\beta}{2}$. We can then write

$$
\omega=\left(\frac{1-\xi^{2}}{1+\xi^{2}}, \frac{2 \xi}{1+\xi^{2}}\right), \quad \sigma=\left(\frac{1-\eta^{2}}{1+\eta^{2}}, \frac{2 \eta}{1+\eta^{2}}\right)
$$

and hence (A.2) reads

$$
x=\frac{-\mu}{1+\xi \eta}(\xi+\eta, \xi \eta-1) .
$$

We can also easily check that we have

$$
t=\mu \frac{\xi-\eta}{1+\xi \eta} .
$$

Summarizing, we have found a correspondence

$$
\begin{gathered}
\mathbb{R}^{2} \backslash\left\{\xi=-\frac{1}{\eta}\right\} \rightarrow \mathbb{R}^{2} \backslash B_{\mu} \\
(\xi, \eta) \rightarrow x_{\xi, \eta}=\frac{-\mu}{1+\xi \eta}(\xi+\eta, \xi \eta-1) .
\end{gathered}
$$

We also note that

- The functions $f$ of the points $x$ in $\mathbb{R}^{2} \backslash B_{\mu}$ are the functions $F$ of the parameters $\omega \in S^{1}$ and $t \in \mathbb{R}$ such that if $x=\mu \omega^{\perp}+t \omega=\mu \sigma^{\perp}-t \sigma$, then $F(\omega, t)=F(\sigma,-t)$.

- The functions $f$ of $x \in \mathbb{R}^{2} \backslash B_{\mu}$ are the functions $F$ of $(\xi, \eta) \in \mathbb{R}^{2} \backslash$ $\left\{\xi=-\frac{1}{\eta}\right\}$ such that $F(\xi, \eta)=F(\eta, \xi)$.

We also remark that

- $f$ extends holomorphically in $t$ if and only if $F$ extends in $\eta$ for fixed $\xi$ or in $\xi$ for fixed $\eta$, 
or in other words

- $f$ extends holomorphically in $t$ if it is separately holomorphic in $\xi$ and $\eta$.

Last, we note that the coordinate transform $(\xi, \eta) \mapsto\left(\xi,-\frac{1}{\eta}\right)$ interchanges the set $\left\{\xi=-\frac{1}{\eta}\right\}$ with the diagonal of $\mathbb{R}^{2}$. Thus Theorem 2.4 above yields at once the proof of the following

Proposition A.1 (Cf. [12, Theorem 3.1]). Let $f(x), x \in \mathbb{R}^{2} \backslash B_{\mu}$ extend as a continuous, separately holomorphic function to the union of the complexifications of the lines tangent to $S_{\mu}$. Then $f$ extends as an entire function to $\mathbb{C}^{2}$.

Proposition A.1 can be applied to the situation that follows. Let $R_{\mu}$ denote the Radon transform defined by

$$
R_{\mu}(f)(\omega, p):=\int_{x \cdot \omega=p} f(x) \mathrm{e}^{\mu x \cdot \omega^{\perp}} d m(x)
$$

where $d m(x)$ is the element of area in the plane $\{x: x \cdot \omega=p\}$. We first recall the fundamental relation between $R_{\mu}$ and the Fourier transform $\mathcal{F}$ which is a consequence of Fubini's Theorem:

$$
\mathcal{F} R_{\mu}(f)(\omega, t)=\mathcal{F}(f)\left(\mathrm{i} \mu \omega^{\perp}+t \omega\right) \quad \text { for any } f \in C_{c}^{\infty} .
$$

Given $F=F(\mathrm{i} \omega, \mathrm{i} t)$ for $(\mathrm{i} \omega, \mathrm{i} t) \in \mathrm{i}\left(\mathbb{R}^{2} \backslash B_{\mu}\right)$ we wonder under what conditions $F$ is the transform $\mathcal{F} R_{\mu}(f)(\omega$, it $)$ of some $f \in C_{c}^{\infty}$. First, according to (A.6) it is necessary that

$$
F(\mathrm{i} \omega, \mathrm{i} t)=F(\mathrm{i} \sigma,-\mathrm{i} t)
$$

whenever $\mu \omega^{\perp}+t \omega=\mu \sigma^{\perp}-t \sigma$. Also, $F$ must be holomorphic in $t$ and endowed with the Paley-Wiener exponential growth estimates in the sense that for some $c^{\prime}$ for any $N$ and for suitable $c_{N}$ we have $|F| \leq c_{N}\left(1+\left|t^{\mathbb{C}}\right|\right)^{-N}$

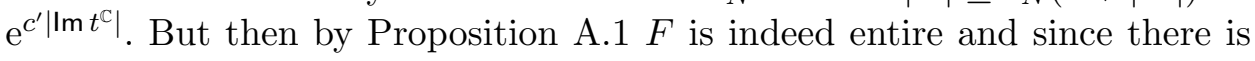
no difficulty in proving that the 2-variables function inherits the exponential growth from the 1-variable one (cf. [12, Th. 3.2]), then it is the Fourier transform of some $f \in C_{c}^{\infty}$ (Paley-Wiener characterization). 
Edge of the wedge theorem for separately holomorphic functions 341

\section{References}

[1] V. Aguilar, L. Ehrenpreis and P. Kuchment, Range condition for the exponential Radon transform, J. Anal. Math. 68 (1996), 1-13.

[2] R.A. Ajrapetyan and G.M. Henkin, Analytic continuation of CR functions through the "edge of the wedge", Sov. Math. Dokl. 24 (1981), 129-132; translation from Dokl. Akad. Nauk SSSR 259 (1981), 777-781.

[3] L. Baracco, A remark on extension of CR functions from hyperplanes, Canad. Math. Bull. 51 (1) (2008), 21-25.

[4] M.S. Baouendi and F. Treves, A property of the functions and distributions annihilated by a locally integrable system of complex vector fields, Ann. Math. 114 (2) (1981), 387-421.

[5] S. Bernstein, Sur l'ordre de la meilleure approximation des fonctions continues par des polynomes de degré donné, Acad. Roy. Belg. Cl. Sci. Mém. 4 (1922), 1-103.

[6] A. Boggess, CR manifolds and the tangential Cauchy-Riemann complex, Studies in Advanced Mathematics, CRC Press Boca Raton, Ann Arbor Boston, London, 1991.

[7] A. Boivin and R. Dwilewicz, Extension and approximation of CR functions on tube manifolds, Trans. Amer. Math. Soc. 350(5) (1998), 1945-1956.

[8] L. Ehrenpreis, The exponential X-ray transform and Fritz John's equation. I. Range description, Contemp. Math. 251 (2000), 173-188.

[9] N. Hanges and F. Treves, Propagation of holomorphic extendability of CR functions, Math. Ann. 263(2) (1983), 157-177.

[10] M. Jarnicki and P. Pflug, An extension theorem for separately holomorphic functions with singularities, Preprint, 2001.

[11] T.V. Nguyen and A. Zeriahi, Familles de polynomes presque partout bornés, Boll. Sci. Math. 107 (1983), 81-91.

[12] O. Öktem, Extension of separately analytic functions and applications to range characterization of the exponential Radon transform, Ann. Polon. Math. 70 (1998), 195-213.

[13] H. Komatsu, A local version of Bochner's tube theorem, J. Fac. Sci. Univ. Tokyo IA 19 (1972), 201-214. 
[14] P. Pflug and V.A. Nguyen, A boundary cross theorem for separately holomorphic functions, Preprint, 2004.

[15] B. Shiffman, Separate analyticity and Hartogs theorems, Indiana Univ. Math. J. 38(4) (1989), 943-957.

[16] J. Siciak, Separately analytic functions and envelopes of holomorphy of some lower dimensional subsets of $\mathbb{C}^{n}$, Ann. Polon. Math. 22 (1970), $145-171$.

[17] J. Siciak, Extremal plurisubharmonic functions in $\mathbb{C}^{n}$, Ann. Polon. Math. 39 (1981), 175-211.

[18] J. Siciak, Extremal plurisubharmonic functionsand capacities in $\mathbb{C}^{n}$, Sophia Kokyuroku in Math, 14, Kyoto Sophia Univ., 01982.

[19] V.P. Zaharjuta, Separately analytic functions, generalizations of Hartog's thorem, and envelopes of holomorphy, Math. Sb. 30 (1976), 51-67.

Dipartimento di Matematica

UNIVERSITA' DI PADOVA

VIA TRIEste 63

35121 PADOVA

ITALY

E-mail address: baracco@math.unipd.it

E-mail address: zampieri@math.unipd.it

Received September 20, 2006 